\title{
Is there a correlation between body mass index and thyroid stimulating hormone?
}

\begin{abstract}
Background: It is believed that overt hyperthyroidism is associated with weight loss and hypothyroidism is associated with weight gain. Studies have shown that there is inter individual variations of thyroid function in persons with thyroid hormone levels within the normal physiological range. A clear epidemiological relation between thyroid hormone status and weight gain and obesity has not been established.
\end{abstract}

Objectives: The study was conducted to determine any correlation between thyroid hormone function indicated by thyroid stimulating factor (TSH) and body mass index (BMI).

Methods: This is cross-sectional study of 316 individuals (26 male and 292 female) out of which 22 individuals had subclinical hypothyroidism ( $\mathrm{SCH}$ ), 208 individuals had hypothyroidism and 88 individuals were euthyroid. TSH was estimated by Chemilluminence immune assay. Anthropometric parameters were measured for all participants and comparisons were done according to gender and thyroid hormone status.

Results: median TSH level was $5.1 \mathrm{mIU}$ for total population, $11.29 \mathrm{mIU}$ in hypothyroid group, $6.86 \mathrm{mIU}$ in subclinical hypothyroid group and $2.60 \mathrm{mIu}$ in euthyroid group. Median BMI was $25.6 \mathrm{~kg} / \mathrm{m}^{2}$ for total population, $25.5 \mathrm{~kg} / \mathrm{m}^{2}$ for hypothyroid group, $26.2 \mathrm{~kg} / \mathrm{m}^{2}$ for subclinical hypothyroid group and $25.6 \mathrm{~kg} / \mathrm{m}^{2}$ for euthyroid group. Median waist circumference was $88.5 \mathrm{~cm}$ for total population, $88.7 \mathrm{~cm}$ for hypothyroid group, $88 \mathrm{~cm}$ for subclinical hypothyroid group and $88 \mathrm{~cm}$ for euthyroid group. $\mathrm{R}^{2}$ for TSH and BMI for hypothyroid group was 0.0003 , for subclinical hypothyroid group was 0.010 and for euthyroid group was 0.006 with $\mathrm{p}$ value being $0.80,0.65$ and 0.45 respectively. $\mathrm{R}^{2}$ for TSH and waist circumference for hypothyroid group was 0.0051 , for subclinical hypothyroid was 0.0002 and for euthyroid was 0.052 with p value being $0.30,0.93$ and 0.03 respectively.

Conclusion: There is no correlation between TSH and BMI. Further studies involving large number of subjects are needed to confirm the findings.

Keywords: body mass index, thyroid, hormone, TSH, BMI, hyperthyroidism, epidemiological, obesity
Volume 7 Issue 5 - 2019

\author{
Ngairangbam Archana, Rajkumari lpsita, Santa \\ Naorem, Lallan Prasad, Salam Ranabir \\ Department of General Medicine, Regional Institute of Medical \\ Sciences, India
}

Correspondence: Salam Ranabir, DM (Endocrinology), Associate Professor, Department of General Medicine, Regional Institute of Medical Sciences, Imphal, India, Email salamranabir@yahoo.co.in

Received: October 09, 2019 | Published: October 31, 2019

\section{Introduction}

According to popular belief overt hyperthyroidism is associated with weight loss ${ }^{1}$ and hypothyroidism is associated with weight gain. ${ }^{2}$ Inter individual variations of thyroid function occur in persons with thyroid hormone levels within the normal physiological range. ${ }^{3}$ Even in individuals with normal thyroid function, recent data has led to the view that an increase in body weight can be due to varied thyroid functions. ${ }^{4,5}$ The Danish Thyroid Study found a positive association between BMI and category of serum TSH and a negative association was found between BMI and category of serum free T4. ${ }^{6}$ Another cross-sectional study it was found that obese euthyroid women had lower T4 levels than did lean euthyroid women. ${ }^{7}$ Other studies showed that serum TSH levels and the grade of obesity had a positive correlation in overweight individuals with a normal thyroid function. ${ }^{8}$

A statistically significant positive correlation was found between BMI and thyroid function in women, while in men the correlation was not so. ${ }^{9} \mathrm{~A}$ community based survey suggested that a positive association exists between TSH and BMI in euthyroid non-smokers. ${ }^{10}$ In healthy adults mean TSH showed an increasing trend with increasing BMI and there was a significant relationship between TSH and increasing BMI. ${ }^{11}$ In contrast to the above some studies have shown no significant relation between body weight or BMI and thyroid function. Beiler et al ${ }^{12}$ concluded that a correlation between BMI and TSH doesn't exist in neither euthyroid nor hypothyroid individuals. Figurosa et $\mathrm{al}^{13}$ stated that there is no association between thyroid hormone function and obesity and weight gain. Neither $\mathrm{T}_{4}$ nor TSH had any correlation with BMI ${ }^{14,15}$ Keeping the above facts in mind it is evident that a clear epidemiological association between thyroid function and body weight has not been established. The purpose of this study is to determine any correlation between thyroid hormone function indicated by TSH and BMI in hypothyroid and subclinical hypothyroid individuals with or without levothyroxine therapy and compare it with euthyroid individuals.

The study was conducted with the following objectives:

i. To determine any correlation between thyroid hormone function indicated by Thyroid Stimulating Hormone and Body Mass Index

ii. To compare BMI in hypothyroid individuals with or without levothyroxine therapy and euthyroid individuals with the respective Thyroid Stimulating Hormone levels. 


\section{Materials and methods}

The study was a cross sectional study conducted in The Department of General Medicine, RIMS, Imphal for 2 years starting from September 2016 to August 2018 with a study population that included euthyroid, hypothyroid or patients with subclinical hypothyroidism irrespective of their treatment status.

Inclusion criteria:

$$
\text { I. Age }-18 \text { to } 65 \text { years }
$$

II. Euthyroid individuals as control

III. Documented or newly diagnosed hypothyroidism (irrespective of treatment status)

IV. Documented or newly diagnosed subclinical hypothyroidism.

Exclusion criteria:

i. Any endocrine disorder other than diabetes with controlled blood glucose for at least 3 months and hypothyroidism

ii. Hyperthyroidism

iii. Pregnant women

iv. Significant renal, liver or heart disease

v. Taking medications that can disrupt thyroid hormone function

Written informed consent was obtained from all the study subjects, prior to the testing of thyroid hormones profile. The subjects underwent a detailed history taking, general physical examination, anthropometric measurements (height, weight, body mass index, waist circumference) and systemic examination. Relevant laboratory investigations were done.

\section{Sample size}

For calculating sample size we utilized correlation value of $r=0.20$ based on a previous study. ${ }^{14}$ A sample size of 194 was calculated based on the above correlation at 5\% significance level with $80 \%$ power. The study was conducted on 318 individuals. The sample size is calculated with the help of an online calculator device owned by University of California and San Francisco (http://www.sample-size. net/correlation-sample-size/).

\section{Working definitions}

Hypothyroidism is defined as low T4 and T3 level with an increased TSH level.

Euthyroid patients is defined as individuals having normal T4, T3 and TSH

Diabetes mellitus is defined as having a diagnosis of diabetes listed in medical history or blood fasting blood glucose levels $\geq 126 \mathrm{mg} \%$ and post prandial blood glucose $\geq 200 \mathrm{mg} \%$.

For diagnosis of hypothyroidism elevated TSH with low T4 was used. ${ }^{17}$

Elevated TSH with normal T4 was taken as subclinical hypothyroidism.

\section{Procedures}

The study was conducted after clearance from the Institute's Ethics Committee. Participants were explained about the purpose and procedure of the study. With the participants consent height, weight and waist circumference were measured and blood samples were taken for measurement of serum TSH level. Test was carried out by Chemilluminence immune assay kit using vitros ${ }^{R}$ ECiQ immunodiagnostic system, Ortho Clinical Diagnostics. Reference range for TSH is $0.465-4.68 \mathrm{mIU} / \mathrm{L}$. BMI calculated according to the formula: Weight $(\mathrm{Kg}) / \operatorname{Height}(\mathrm{m})^{2}$

\section{Statistical analysis}

The data obtained from the participants were expressed as median and range and comparison of median between the different groups was done using simple linear regression equation and one way analysis of variance. Microsoft excel 2016 was used for analysis of the collected data. A $p$ value of less than 0.05 was considered significant.

\section{Results}

This study included a total of 318 individuals (26male and 292female) out of which 22 individuals had subclinical hypothyroidism (SCH), 208 individuals had hypothyroidism and 88 individuals were euthyroid. Female preponderance was seen as 292 out of $318(91.823 \%)$ participants were female. Median TSH level was $5.1 \mathrm{mIU}$ for total population, $11.29 \mathrm{mIU}$ in hypothyroid group, $6.86 \mathrm{mIU}$ in subclinical hypothyroid group and $2.60 \mathrm{mIu}$ in euthyroid group. Median BMI was $25.6 \mathrm{~kg} / \mathrm{m}^{2}$ for total population, $25.5 \mathrm{~kg} / \mathrm{m}^{2}$ for hypothyroid group, $26.2 \mathrm{~kg} / \mathrm{m}^{2}$ for subclinical hypothyroid group and $25.6 \mathrm{~kg} / \mathrm{m}^{2}$ for euthyroid group. Median waist circumference was $88.5 \mathrm{~cm}$ for total population, $88.7 \mathrm{~cm}$ for hypothyroid group, $88 \mathrm{~cm}$ for subclinical hypothyroid group and $88 \mathrm{~cm}$ for euthyroid group as shown in Table $1 . \mathrm{R}^{2}$ for TSH and BMI for hypothyroid group were 0.0003 , for subclinical hypothyroid group were 0.010 and for euthyroid group were 0.006 with $\mathrm{p}$ value being $0.80,0.65$ and 0.45 respectively (Table 2 ). $\mathrm{R}^{2}$ for TSH and waist circumference for hypothyroid group was 0.0051 , for subclinical hypothyroid was 0.0002 and for euthyroid was 0.052 with p value being $0.30,0.93$ and 0.03 respectively as shown in Table 3. The above data showed that there is no significant correlation between TSH with BMI or waist circumference in hypothyroid, euthyroid or subclinical hypothyroid individuals.

Table I Anthropometric parameters and TSH according to thyroid status

\begin{tabular}{lllll}
\hline Parameters & Total & Hypothyroid & Subclinical & Euthyroid \\
\hline Median (Range) & $(\mathrm{n}=318)$ & $(\mathrm{n}=208)$ & $(\mathrm{n}=22)$ & $(\mathrm{n}=88)$ \\
Age (in years) & $36(17-65)$ & $37(17-65)$ & $36(21-63)$ & $36(18-65)$ \\
Weight (in kg) & $60(37-98)$ & $60(37-95)$ & $62.5(42-90)$ & $60(44-98)$ \\
BMI $(\mathrm{kg} / \mathrm{m} 2)$ & $25.6(17.7-37.1)$ & $25.5(17.7-37.1)$ & $26.2(21.4-35.7)$ & $25.6(19.0-37.0)$ \\
WC $(\mathrm{cm})$ & $88.5(60-164)$ & $88.7(62-163)$ & $88(60-107)$ & $88(60-164)$ \\
TSH $(\mathrm{mlU} / \mathrm{L})$ & $5.1(0.01-321.2)$ & $11.29(0.01-321.2)$ & $6.86(4.6-9)$ & $2.60(0.43-4.5)$ \\
\hline
\end{tabular}

$\mathrm{BMI}$ - body mass index;WC - waist circumference; TSH - thyroid stimulating hormone 
Table 2 Coefficient of variation between different groups of thyroid status and $\mathrm{BMI}$

\begin{tabular}{llll}
\hline & Hypothyroid & Subclinical & Euthyroid \\
\hline $\mathrm{R}^{2}$ & 0.0003 & 0.01 & 0.006 \\
$\mathrm{P}$ value & 0.8 & 0.65 & 0.45
\end{tabular}

Table 3 Coefficient of variation between different groups of thyroid status and waist circumference

\begin{tabular}{lllll}
\hline & Total & Hypothyroid & Subclinical & Euthyroid \\
\hline $\mathrm{R}^{2}$ & 0.0035 & $0.005 \mathrm{I}$ & 0.0002 & 0.052 \\
$\mathrm{P}$ value & 0.28 & 0.303 & 0.939 & 0.03 \\
\hline
\end{tabular}

\section{Discussion}

The aim of this study was to determine any correlation between thyroid hormone function and body mass index. Our study included 318 individuals, 22 with subclinical hypothyroidism, 208 with hypothyroidism and 88 with euthyroid status. There was a clear female preponderance in all the three groups of patients with female constituting 292 out of 318 (91.82\%) individuals who participated. Similar to study conducted by Meng et al. ${ }^{19}$ females had a higher incidence of thyroid dysfunction than males along with a higher BMI. Similar to a study conducted by Anderson et $\mathrm{al}^{3}$ we found a wide variation in the TSH value in healthy euthyroid subjects. Our patients were divided into three groups according to their thyroid hormone status after fulfilling all the inclusion criteria namely hypothyroid group, subclinical hypothyroid group and euthyroid group. The median age, weight and BMI were comparable in all the three groups. This is contradictory to the study conducetd by Michalaki et $\mathrm{al}^{20}$ in which they concluded that prevalence of overt and subclinical hypothyroidism was higher in obese subjects. From this we can infer that although there is a significant difference between the TSH values in the 3 groups, the difference between the weight and BMI is insignificant. These findings were similar to a studies conducted by Bieler et al. ${ }^{17}$ and Figurosa et al. ${ }^{16}$ in which they concluded that there was no significant relationship between BMI and TSH of neither hypothyroid nor euthyroid individuals and no association between thyroid hormone status and obesity or weight gain. Similar findings were also noted by Akshoy et al, ${ }^{21}$ in a study of BMI in euthyroid and subclinical hypothyroid individuals where they found no difference in the BMI between the two groups. Manji et al.,22 also stated that there is no difference between TSH/fT $\mathrm{T}_{4}$ in obese and lean subjects.

Nakamura et al, ${ }^{23}$ concluded that there was no correlation between percentage body fat and serum leptin (which is increased in hypothyroid individuals) in euthyroid and hyperthyroid individuals. Bjerved et $\mathrm{al}^{11}$ suggested that TSH levels are not a determinant of future weight changes and BMI is not a determinant of TSH changes. Similar to this study our results also suggest that there might not be any benefit with regard to body weight in keeping TSH in the lower half of the normal range. On plotting our findings on a scatter diagram by taking TSH as independent variable and BMI as dependent variable and applying simple linear regression equation we found no correlation between TSH and BMI in all the three groups in both males and females which is contrary to what was suggested by Asvold et al. ${ }^{13}$ and Sakurai et al..$^{24}$ There are several studies that indicate that weight gain is associated with increase in $\mathrm{TSH}^{4-10}$ In a retrospective study conducted by Bastemir M et al., ${ }^{8}$ on 226 euthyroid obese or overweight females they found that serum TSH were higher in the obese than in the lean individuals, but it is not clear whether the reverse is true.
While some other studies have suggested that TSH and BMI have a significant association within the normal $\mathrm{TSH}$ range ${ }^{4,13}$ our study which also include healthy individuals with normal TSH did not show any such association and in fact there was no significant difference between the BMI of the 3 study population. As opposed to a study which concluded that hyporthyoidism is associated with an increase in BMI and serum leptin levels, ${ }^{25}$ we did not find a significant difference between the BMI in hypothyroid, euthyroid and subclinical hypothyroid individuals. We also noticed that although the incidence of thyroid hormone disorders are more in females than in males there was no correlation between TSH and BMI in both males and females irrespective of their thyroid hormone status. This finding is contradictory to a study conducted by Millionis et $\mathrm{al}^{9}$ in which correlation between BMI and thyroid function was statistically significant in women and another study conducted by Pergola et al. ${ }^{26}$ where they suggested a relation between body weight, waist circumference and thyroid hormone function. Although there is well known association between energy expenditure, thermogenesis and thyroid function ${ }^{27}$ and lower serum T3 is associated with decreased resting metabolic rate ${ }^{28}$ the possible role of diet and lifestyle were not assessed. We also measured waist circumference in all the subjects and we noticed that the median and range of waist circumference were similar in the three groups. There was no correlation between TSH and waist circumference in all the three groups in our study irrespective of their thyroid hormone status. This finding is similar to a study conducted by Kitahara et al. ${ }^{15}$ in which they concluded that there is no significant interaction between BMI or waist circumference with $\mathrm{TSH}, \mathrm{fT}_{3}$ and $\mathrm{fT}_{4}$. Measurement of $\mathrm{fT}_{3}$ and $\mathrm{fT}_{4}$ and comparing it with waist circumference would perhaps confirm this finding. Our results contradict the arguments for a causal relationship between TSH and BMI. Further prospective analysis where follow up data is available may help to confirm or refute our findings.

The strengths of this study include a reasonably large sample size that includes euthyroid controls, subclinical and hypothyroid patients. We excluded patients with hyperthyroidism and those with diseases or disorders or on drugs that may have any impact on weight and BMI. Diabetic patients with poor blood sugar control were also excluded and those whose blood glucose was controlled for atleast 3 months were included. We also excluded individuals who are in the extreme age groups as old age itself can lead to weight gain and increase in BMI due to reduced resting metabolic rate. ${ }^{29}$ Our study also has major weaknesses. Firstly, the observational design of the study with no follow up data available. Secondly, although $\mathrm{T}_{4}$ was measured for diagnosis of thyroid disorder with $\mathrm{TSH}$, free $\mathrm{T}_{3}$ and free $\mathrm{T}_{4}$ were not measured, which could have added additional information on the subject's thyroid hormone status. Thirdly, lifestyle, dietary habits and exercise were not considered which could act as independent confounding factor and which could play a major role in TSH receptor expression in abdominal adipose tissue ${ }^{30,31}$

\section{Conclusion}

We conclude that there is no association between thyroid stimulating hormone and body mass index. And that there is unlikely to be any benefit in maintaining TSH in the lower half of the normal range with regard to weight loss. Other options like dietary changes, lifestyle modification and exercise should be advised instead.

\section{Acknowledgments}

None. 


\section{Conflicts of interest}

The authors declare that there is no conflict of interest.

\section{Funding}

None.

\section{References}

1. Hoogwerf BJ, Nuttall FQ. Long-term weight regulation in treated hyperthyroid and hypothyroid subjects. Am J Med.1984;76(6):963-970.

2. Baron DN. Hypothyroidism; its aetiology and relation to hypometabolism, hypercholesterolaemia and increase in body weight. Lancet. 1956;271(6937):277-281.

3. Anderson S, Pederson KM, Bruun NH, et al. Narrow individual variations in serum $\mathrm{T} 4$ and $\mathrm{T} 3$ in normal subjects:A clue to the understanding of subclinical thyroid disease. J Clin Endocrinol Metab. 2002;87(3):1068-1072.

4. Nyrnes A, Jorde R, Sundsfjord J. Serum TSH is positively associated with BMI. International journal of obesity. 2006;30(1):100-105.

5. Fox CS, Pencina MJ, D’Agostino RB, et al. Relations of thyroid function to body weight. Arch Intern Med. 2008;168(6):587-592.

6. Knudson N, Laurberg P, Rasmussen LB, et al. Small differences in thyroid function may be important for body mass index and the occurrence of obesity in the population. $J$ Clin Endocrinol Metab. 2005;90(7):4019-4024.

7. Shon HS, Jung ED, Kim SH, et al. Free T4 is negatively correlated with body mass index in euthyroid women. Korean J Intern Med. 2008;23(2):53-57.

8. Bastemir M, Akin F, Alkis E, et al. Obesity is associated with increased serum TSH level, indepent of thyroid function. Swiss Medical Weekly. 2007;137(29-30):431-434.

9. Milionis A, Milionis C. Correlation between body mass index and thyroid function in euthyroid individuals in Greece. Hindawi J. 2013;651494.

10. Mehran L, Amouzegar A, Delshad H, et al. Association between serum TSH concentration and body mass index in euthyroid subjects:the role of smoking. Archives of Iranian Medicine. 2012;15(7):400-403.

11. Solanki A, Bansal S, Jindal S, et al. Relationship of thyroid stimulating hormone with body mass index in healthy adults. India $j$ of Endocrinol Metab. 2013;17(1):167-169.

12. Bieler BM, Gaugham J, Khan M, et al. Lack of an association between BMI and TSH in treated hypothyroid patients and euthyroid controls. Endocr Pract. 2016;22(5):555-560.

13. Figueroa B, Velez H, Ramirez MI. Association of thyroid stimulating hormone levels and body mass index in overweight Hispanics in Puerto Rico. Ethn Dis. 2008;18[Suppl 2]:S2-151-154.

14. Hashimoto K, Tagami T, Yamakage H, et al. Serum free thyroxine levels are associated with the efficacy of weight reduction therapy in obese female patients. Endocrine Journal. 2016;63(3):221-229.

15. Kitahara CM, Platz EA, Ladenson PW, et al. Body fatness and markers of thyroid function among U.S. men and women. Plos one. 2012;7(4):e34979.

16. Asvold BO, Bjoro T, Vatten LJ. Association of serum TSH with high body mass differs between smokers and never smokers. Journal of Clin Endocrinol Metab. 2009;94(12):5023-5027.

17. Sakurai M, Nakamura K, Miura K, et al. Association between a serum thyroid stimulating hormone concentration within the normal range and indices of obesity in Japanese men and women. Intern Med. 2014;53(7):669-674.

18. Garber JR, Cobin RH, Gharib H, et al. Clinical practice and guidelines for hypothyroidism in adults:Cosponsored by The American Association of Clinical Endocrinologists and The American Thyroid Association. Thyroid. 2012;22:12.

19. Roos A, Bakker SJL, Links TP, et al. Thyroid function is associated with components of the metabolic syndrome in euthyroid subjects. The Journal of Clinical Endocrinology and Metabolism. 2007;92(2):491-496.

20. Aksoy N, Yeler MT, Ayan NN, et al. Association between thyroid hormone levels and insulin resistance and body mass index. Pak J Med Sci. 2015;31(6):1417-1420.

21. Pergola G, Nardecchia A, Ammirati A, et al. Abdominal obesity is characterized by higher pulse pressure: Possible role of free triiodothyronine. Journal of obesity. 2012;2012:656303.

22. Lu S, Guan Q, Liu Y, et al. Role of extrathyroid TSHR expression in adipocyte differentiation and its association with obesity. Lipids in Health and Disease. 2012;11:17.

23. Bjergved L, Jorgensen $\mathrm{T}$, Perrild $\mathrm{H}$, et al. Thyroid function and body weight:A community based longitudinal study. Plos one. 2014;9(4):e93515.

24. Zhang Y, Lu P, Zhang L, et al. Association between lipids profile and thyroid parameters in euthyroid diabetic subjects: a cross sectional study. BMC Endocr Disord. 2015;15:12.

25. Bell A, Gagnon A, Grunder L, et al. Functional TSH receptor in human abdominal preadipocytes and orbital fibroblasts. Am J Physiol. 2000;279(2):C335-C440.

26. Chen MD, Song YM, Tsou CT, et al. Leptin concentration and the $\mathrm{Zn} / \mathrm{Cu}$ ratio in plasma in women with thyroid disorder. Biological trace element research. 2000;75(1-3):99-105.

27. Nakamura T, NagasakaS, IshikawaS, etal.Association of hyperthyroidism with serum leptin levels. Metabolism. 2000;49(10):1285-1288.

28. Dale J, Daykin J, Holder R, et al. Weight gain following treatment of hyperthyroidism. Clin Endocrinol. 2001;55(2):233-239.

29. Michalaki MA, Vagenekis AG, Leonardou AS, et al. Thyroid function in humans with morbid obesity. Thyroid. 2006;16(1):73-78.

30. Manji N, Boelaert MC, Holder RL, et al. Lack of association between serum TSH or free $\mathrm{T}_{4}$ and body mass index in euthyroid subjects. Clin Endocrinol. 2006;64(2):125-128.

31. Brunova $\mathrm{J}$, Bruna $\mathrm{J}$, Joubert $\mathrm{G}$, et al. Weight gain in patients after therapy for hyperthyroidism. S Afr Med J. 2003;93(7):529-531.

32. Sari R, Balci MK, Altunbas H, et al. The effect of body weight and weight loss on thyroid volume and function in obese women. Clin Endocrinol. 2003;59(2):258-262.

33. Meng Z, Liu M, Zhang Q, et al. Gender and age impacts on association between thyroid function and metabolic syndrome in Chinese. Medicine. 2015;94(50):e2193.

34. Silva JE. Thyroid hormone control of thermogenesis and energy balance. Thyroid. 1995;5(6):481-492.

35. Astrup A, Buemann B, Toubro S, et al. Low resting metabolic rate in subjects predisposed to obesity:a role of thyroid status. Am J Clin Nutr. 1996;63(6):879-883.

36. Stonge MP, Gallagher D. Body composition changes with aging: the cause or the result of alterations in metabolic rate and macronutrient oxidation. Nutrition. 2010;26(2):152-155. 